\title{
Conditions actuelles de la maîtrise de la reproduction en France
}

\author{
H Leridon
}

\author{
INED, 27, rue du Commandeur, 75675 Paris Cedex 14, France
}

(28 Réunion de la Société Française pour l'Étude de la Fertilité; Paris, 19-21 octobre 1989)

\begin{abstract}
Résumé - La fécondité française a fortement baissé au cours des années 1965-1976, essentiellement par diminution des naissances de rang 3 et plus. Cette évolution résulte à la fois d'une baisse du nombre d'enfants souhaité par les couples, et d'une forte réduction des naissances non désirées. Une enquête nationale réalisée par l'INED en 1988 met en évidence la diffusion rapide des méthodes contraceptives médicalisées (pilule et stérilet) depuis 1965. Dans cette même enquête, une proportion élevée de femmes a déclaré avoir eu des difficultés pour concevoir : ces difficultés se sont traduites par un allongement très sensible du temps nécessaire pour concevoir, même si l'impatience des femmes à cet égard semble, elle aussi, croissante.
\end{abstract}

fécondité / contraception / stérilité / naissances non désirées

Summary - Present control of reproduction in France. Fertility declined rapidly in France between 1965 and 1976, mainly because of a decrease in the number of births of parity 3 or more. This change was due to both a decline in the desire for children and to a large reduction in the number of unwanted births. The INED national survey of 1988 showed a rapid spread of medical contraceptive techniques (pill and IUD) since 1965. In the same survey, a significant proportion of women reported difficulties in conceiving: these difficulties resulted in a much longer time-interval before conception; in addition women tended to become more and more impatient in this regard.

fertility / contraception / sterility / unwanted births

La fécondité française est sensiblement constante depuis une douzaine d'années. L'indicateur conjoncturel (global) varie très peu, et la descendance finale des générations - déjà connue pour les générations antérieures à 1945, estimée pour les générations 1945 à 1954 - semble de stabiliser entre 2,0 et 2,1 enfants par femme, c'est-à-dire au voisinage du "seuil de remplacement» des générations. Cette évolution récente est à resituer dans une rétrospective plus longue (figure 1) (Leridon et al, 1987). Dans la société traditionnelle des XVIIe et XVIIIe siècles, une Française encore en vie vers son cinquantième anniversaire avait mis au monde, en moyenne, 5 à 6 enfants, dont plus de la moitié décédaient avant l'âge adulte. Dès la seconde moitié du XVIIIe siècle, la fécondité a commencé à fléchir, pour atteindre son minimum historique avec les dernières générations du XIXe siècle : 2,0 enfants par femme exactement, dans un contexte de mortalité encore assez élevé. Le babyboom a fait remonter la moyenne audessus de 2,6 (générations 1930-1932), 


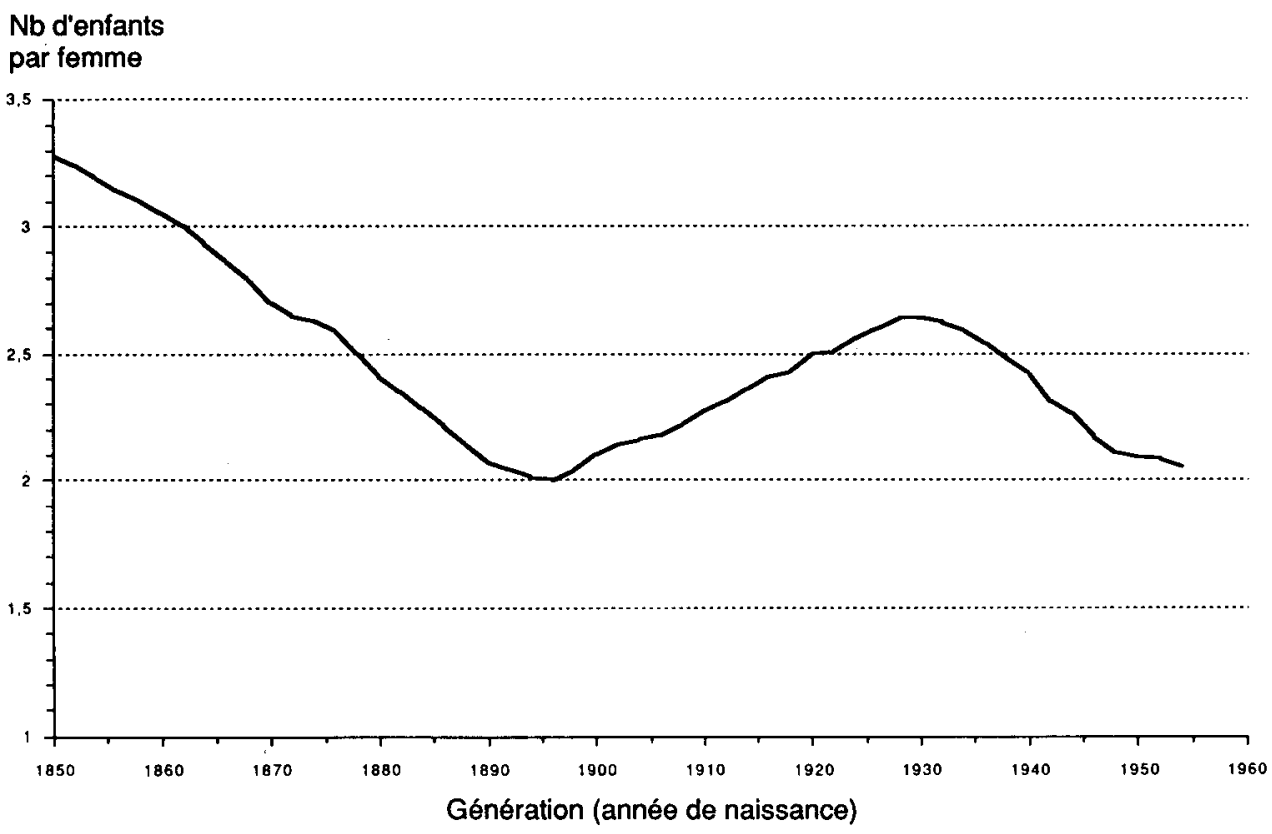

Fig 1. Evolution de la descendance finale des générations en France (1850-1955).

et l'on est revenu en une vingtaine d'années autour de 2,1 enfants par femme (générations 1950-1954).

La baisse de la période 1965-1975 a une caractéristique importante : elle n'a porté, à très peu près, que sur les naissances de rangs 3 et plus (figure 2) : $88 \%$ des femmes des générations 1930-1932 ont eu au moins un enfant, et $70 \%$ en ont eu au moins deux; il en sera exactement de même pour la génération 1955. En revanche, si 100 femmes du premier groupe ont eu en moyenne 104 enfants de rang 3 ou plus, 100 femmes du second groupe n'en auront que 45 , soit à peine la moitié.

II y a donc eu une modification rapide et substantielle des comportements. Cette évolution peut avoir deux origines, d'ailleurs non indépendantes : soit une révision à la baisse du nombre d'enfants dé- siré, soit une meilleure maîtrise de la reproduction grâce à la contraception et à l'avortement. Les deux causes, on va le voir, ont joué, et on peut leur attribuer un poids à peu près égal.

Le nombre idéal d'enfants est un indicateur de l'intensité du désir d'enfants. Il était voisin de 2,8 avant 1965, et il est tombé à 2,5 après 1975. Plus significatif, sans doute, est l'idéal «situé dans son milieu social», généralement un peu inférieur au précédent, et il est préférable de le limiter aux 25-34 ans, c'est-à-dire à la période de la vie durant laquelle se constitue la plus grande partie de la descendance. Cet indicateur était proche de 2,5 avant 1965 , et il est compris entre 2,2 et 2,3 depuis 1975 . Comme on le voit sur la figure 3 , son évolution a donc été moins rapide que celle de la fécondité effective, et elle "n'explique» 


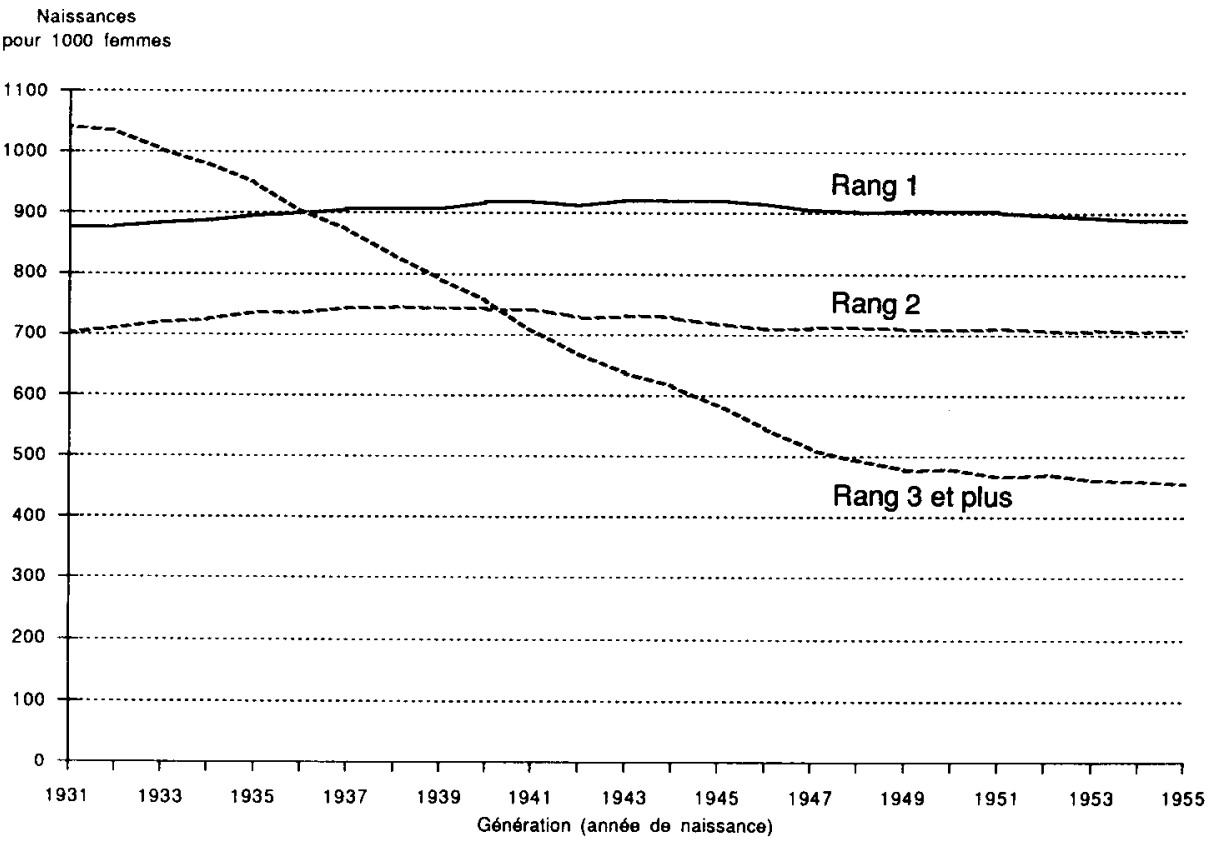

Fig 2. Evolution de la descendance par rang de naissance (générations 1931-1955).

qu'une moitié environ de la baisse de celle-ci. L'analyse des naissances non désirées va nous permettre d'expliquer l'autre moitié.

La maîtrise de la reproduction, en effet, n'est jamais parfaite. D'une part, aucune des méthodes contraceptives disponibles actuellement n'a une efficacité absolue, surtout en termes "d'efficacité d'usage", c'est-à-dire compte tenu des inévitables aléas de la vie courante. D'autre part, la motivation présidant à la mise en cuvre de ces méthodes est d'intensité variable selon les individus, les étapes du cycle de vie ou les circonstances de la vie amoureuse. Des «échecs" se produisent donc plus ou moins fréquemment, mais ce qu'on peut attendre à la fois d'une diminution du nombre d'enfants désirés et de la rapide diffusion des méthodes contraceptives les plus modernes, c'est une réduction du nombre de ces échecs. II en a bien été ainsi.

Pour le vérifier, I'INED a interrogé à deux reprises un large échantillon de femmes sur l'ensemble de leurs grossesses antérieures, en demandant à chacune de préciser si cjuste avant la grossesse, elle souhaitait être enceinte : à ce moment, plus tard, plus tôt ou pas du tout ?". Il est, d'abord, intéressant de constater que les réponses obtenues rétrospectivement dans chacune des deux enquêtes pour les naissances d'une même période sont concordantes : autrement dit, le jugement que l'on peut porter sur les circonstances d'une grossesse n'est pas altéré par le temps, il reste le même 5 ans après l'événement ou 15 ans après.

La figure 4 mantre que la diminution du nombre de "naissances non désirées" (réponse "pas du tout») et celle du nombre 


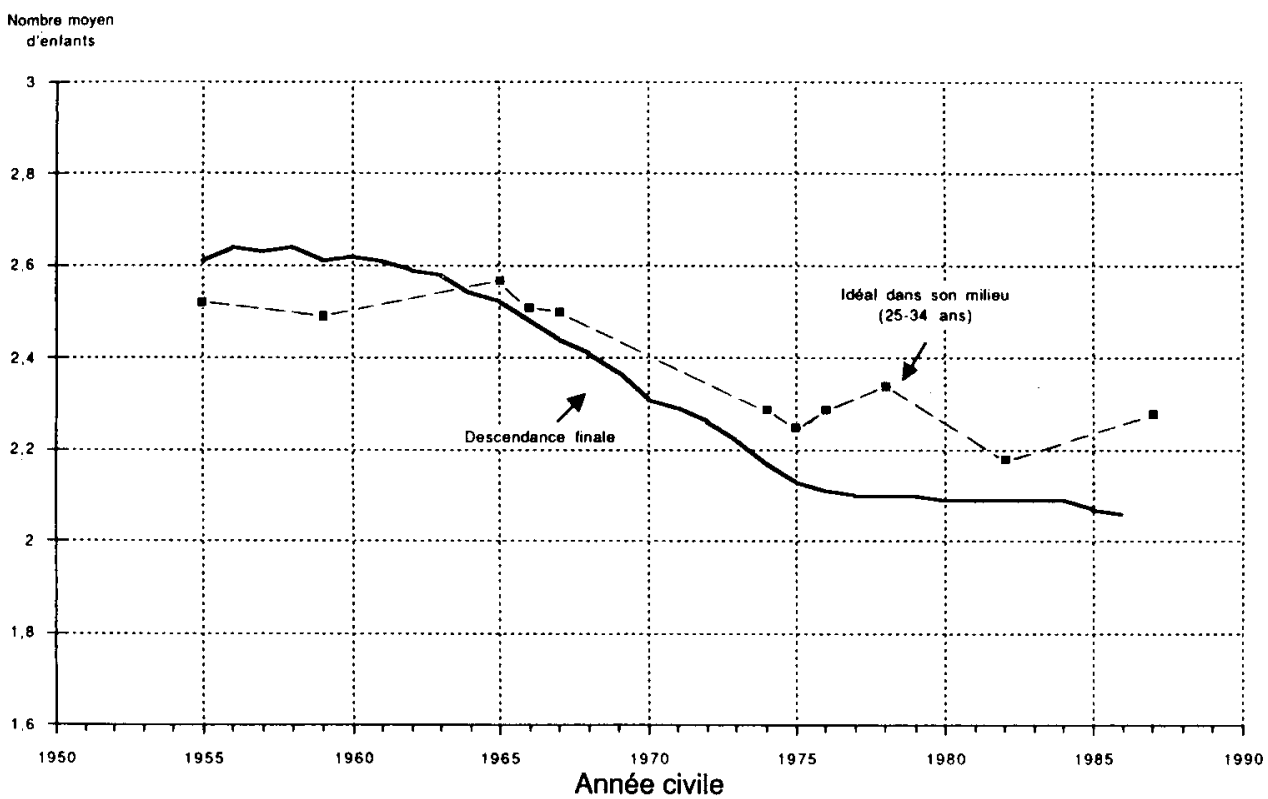

Fig 3. Evolution du nombre idéal d'enfants et de la fécondité (1955-1987). La descendance finale de l'année $\mathrm{A}$ est celle de la génération née 27 ans avant l'année $\mathrm{A}$.

de «naissances mal programmées» (réponse "plus tard") ont été rapides entre 1965 et 1980 (environ). On comptait en début de période : 0,59 naissance non désirée et 0,61 mal programmée, par femme, pour 2,83 naissances en tout (soit, respectivement, 21 et $22 \%$ de la fécondité totale); et en fin de période (vers 1985) : 0,17 naissance non désirée et 0,20 mal programmée, pour 1,82 en tout (soit 9 et $11 \%$ de la fécondité totale).

L'analyse par génération montre des tendances comparables : les cohortes 1953-1957 retrouvent même une fécondité "correctement planifiée" identique à celle des cohortes 1933-1937 (1,55 enfant par femme), alors que la descendance finale aura chuté de 0,5 enfant $(-20 \%)$. II est donc clair que le baby-boom des années cinquante a été constitué, pour une large part, de naissances qui n'étaient pas vraiment souhaitées, ou qui sont survenues plus tôt que prévu dans la vie des couples. Le niveau auquel est descendue aujourd'hui la fécondité résulte de la quasidisparition de ces naissances.

Par quels moyens est-on parvenu à ce résultat ? L'avortement naturellement a pu jouer son rôle. Mais il faut rappeler que le nombre d'IVG enregistré (et même celui estimé en tenant compte d'un sousenregistrement possible) depuis 1976 n'est guère différent de celui des avortements clandestins avant cette date, tel qu'on avait pu l'estimer par des voies indirectes. Mais les nouvelles méthodes contraceptives ont, surtout, eu leur part (Leridon et Toulemon, 1988).

Sur 100 femmes âgées de 20 à 44 ans, 5 utilisaient la pilule ou le stérilet en 1968 , 


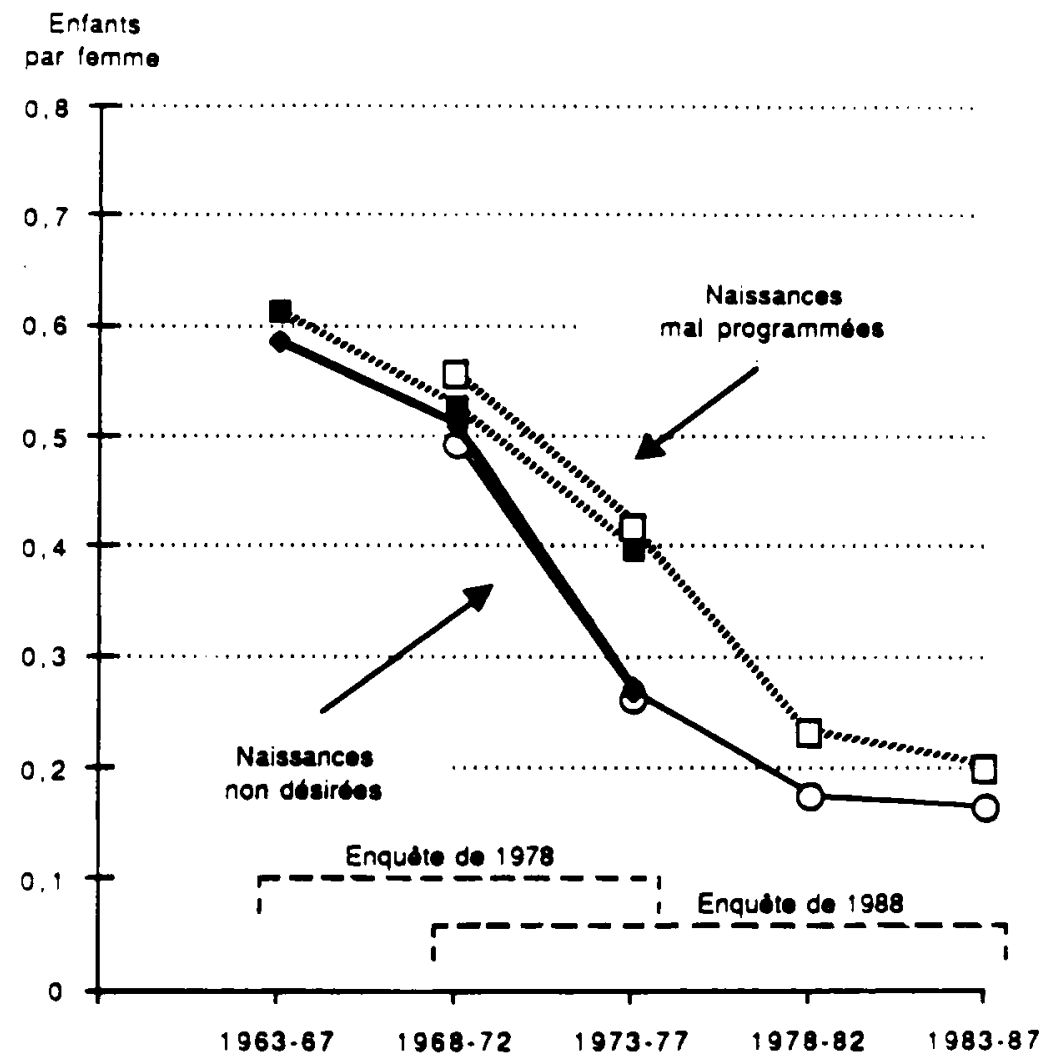

Flg 4. Evolution de la fécondité non désirée et de la fécondité mal programmée (en naissances par femme, 1963-1987).

36 en 1978, 52 en 1988; parallèlement, les méthodes "traditionnelles" ont fortement reculé, ne concernant plus qu'à peine $10 \%$ des femmes de 20-44 ans en 1988. Le tableau I détaille les méthodes utilisées actuellement selon l'âge, et les motifs de non-utilisation. Pour la pilule, le maximum d'utilisation est atteint dès 20-24 ans (51\% des femmes de ce groupe d'âge), pour le stérilet vers 35-39 ans. II y a clairement substitution progressive, quand l'âge augmente, d'une méthode à l'autre : la pilule est très fréquemment utilisée par les femmes les plus jeunes, au point qu'à 20 ans une femme sur deux y a déjà eu recours, et à 30 ans près de neuf sur dix sont dans ce cas; le stérilet prend progressivement le relais entre 30 et 45 ans, et dépasse la pilule à partir de 35 ans.

Ces chiffres révèlent aussi un aspect important de la contraception en France : sa très forte médicalisation. Les deux méthodes principales, en effet, ont en commun de requérir une prescription médicale et un suivi régulier, soit pour renouveler la prescription initiale (pilule), soit pour véri- 
Tableau I. Méthodes contraceptives utilisées en France (Enquête INED, 1988).

Proportion (\%)

de femmes qui :
Age au $1^{\text {er }}$ janvier 1988 (ans)

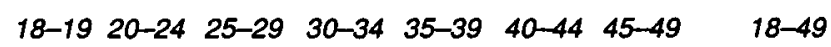

\begin{tabular}{|c|c|c|c|c|c|c|c|c|}
\hline $\begin{array}{l}\text { Utilisent une } \\
\text { méthode : } \\
\text { dont : }\end{array}$ & 51,4 & 60,4 & 69,8 & 73,6 & 71,6 & 62,3 & 47,6 & 64,4 \\
\hline pilule & 44,4 & 50,8 & 41,7 & 33,5 & 25,0 & 14,2 & 11,0 & 31,8 \\
\hline stérilet & 0,4 & 2,7 & 14,9 & 25,6 & 27,8 & 25,3 & 14,2 & 17,3 \\
\hline préservatifs & 2,5 & 2,0 & 4,3 & 4,2 & 4,9 & 5,9 & 3,0 & 4,0 \\
\hline retrait & 2,5 & 2,0 & 4,8 & 6,5 & 7,3 & 11,6 & 15,1 & 7,0 \\
\hline abstinence périodique & 0,8 & 1,3 & 1,9 & 1,3 & 3,6 & 4,3 & 3,1 & 2,4 \\
\hline autres (ou inconnues) & 0,8 & 1,6 & 2,2 & 2,5 & 3,0 & 1,0 & 1,2 & 1,9 \\
\hline \multicolumn{9}{|l|}{ N'utilisent pas } \\
\hline $\begin{array}{l}\text { de méthode : } \\
\text { dont : }\end{array}$ & 48,6 & 39,6 & 30,3 & 26,4 & 28,4 & 37,6 & 52,4 & 35,6 \\
\hline stérilisation & _- & 0,2 & 0,2 & 4,1 & 9,4 & 15,9 & 21,2 & 7,1 \\
\hline stériles & - & 0,2 & 1,3 & 1,1 & 3,9 & 7,5 & 18,8 & 4,2 \\
\hline enceintes & 0,8 & 8,3 & 10,2 & 5,6 & 2,1 & 1,0 & - & 4,7 \\
\hline sans partenaire & 47,0 & 25,1 & 8,1 & 7,5 & 7,4 & 6,9 & 9,7 & 13,3 \\
\hline $\begin{array}{l}\text { et : } \\
\text { veulent }\end{array}$ & & & & & & & & \\
\hline \multirow{2}{*}{$\begin{array}{l}\text { veulent } \\
\text { des enfants } \\
\text { ne veulent plus } \\
\text { d'enfant }\end{array}$} & 0,8 & 5,6 & 9,5 & 6,5 & 3,7 & 0,8 & 0,4 & 4,5 \\
\hline & - & 0,2 & 1,0 & 1,6 & 1,9 & 5,5 & 2,3 & 1,8 \\
\hline Ensemble & 100 & 100 & 100 & 100 & 100 & 100 & 100 & 100 \\
\hline $\begin{array}{l}\text { Population totale } \\
\text { (milliers) }\end{array}$ & 811 & 2096 & 2086 & 2094 & 2144 & 1757 & 1384 & 12372 \\
\hline
\end{tabular}

Source : Leridon et Toulemon, 1988.

fier que tout se passe bien (stérilet). En pratique, une consultation tous les six mois semble nécessaire. Or dans la plupart des autres pays à forte pratique contraceptive, la stérilisation tient une place nettement plus importante qu'en France, spécialement au Royaume-Uni, aux Pays-Bas, aux Etats-Unis et au Canada. Certes, la stérilisation est un acte médical, mais elle n'implique pas ultérieure- ment un suivi aussi régulier que les méthodes réversibles.

Si environ une femme (d'âge reproductif) sur trois n'utilise aucune méthode, il y a à cela quelque raison. Bon nombre d'entre elles, tout d'abord, ne sont probablement pas "au risque " d'une grossesse non désirée, faute de partenaire (mari, concubin, ou tout autre "partenaire régulier»). Cer- 
taines $(7 \%$ des femmes de $18-49$ ans, mais $16 \%$ de celles de $40-44$ ans) ont subi une opération stérilisante, pas forcément à but contraceptif; ces chiffres sont plutôt en recul par rapport à 1978. D'autres sont stériles (nous y reviendrons) ou enceintes (volontairement !) ou à la recherche d'une grossesse. Ne restent finalement que moins de $2 \%$ des "cas inexpliqués" pour lesquels il pourrait y avoir exposition au risque d'une grossesse non voulue sans protection apparente.

Si les couples se protègent donc de mieux en mieux contre ce type de risque, en est-il de même pour le risque inverse, c'est-à-dire les difficultés à concevoir ? Là encore, la comparaison entre les deux enquêtes nationales de l'INED (1978 et 1988) nous apporte des éléments de réponse précieux, quoique ici plus difficiles à interpréter.

Le constat brut de départ est le suivant : sur 100 femmes âgées de 25 à 44 ans et ayant cherché à concevoir : $6 \%$ ont déclaré (en 1988) ne pas y être parvenues, au moins une fois; $25 \%$ ont déclaré uavoir mis plus de temps qu'elles ne l'auraient voulu pour être enceintes".

Le premier chiffre n'est pas un indicateur de "stérilité totale", puisque $40 \%$ des femmes de ce groupe ont eu au moins un enfant, avant de se heurter à une impossibilité de commencer à nouveau une grossesse. C'est donc moins de $4 \%$ des femmes qui resteraient involontairement sans aucun enfant.

Le second chiffre ne constitue pas un indicateur objectif d'shypofertilité", puisqu'il ne reflète que l'appréciation (éventuellement subjective) des intéressées. Cellesci étaient d'ailleurs invitées à préciser "combien de temps elles avaient dû attendre pour devenir enceintes». La réponse moyenne dépasse 21 mois, ce qui est indiscutablement un délai très supé- rieur à la normale. Mais $25 \%$ ont déclaré un délaj inférieur ou égal à 6 mois, $35 \%$ un délai strictement inférieur à 12 mois, et $56 \%$ un délai inférieur ou égal à 12 mois. Si l'on s'en tient donc à la définition la plus courante de la "stérilité", expression d'ailleurs impropre et qu'il faudrait remplacer par l' "hypofertilité", il faut exclure de la catégorie déclarant des difficultés toutes les femmes ayant conçu en 12 mois ou moins; il n'en reste plus alors que $11 \%$, au lieu de $25 \%$.

En 1978, le nombre de questions posées était plus limité, ce qui rend délicates les comparaisons. La question initiale était la même, et le constat brut est celui d'une forte hausse des difficultés déclarées entre 1978 et $1988(+70 \%)$. Mais on doit immédiatement se demander si ce n'est pas surtout la sensibilité au problème qui a augmenté, et si les couples ne sont pas simplement plus impatients et exigeants aujourd'hui qu'hier. Deux éléments de nos enquêtes vont dans ce sens.

Le premier résulte de la comparaison des répartitions de l'ancienneté de l'attente pour les femmes déclarant des difficultés au moment de l'enquête. La figure 5 montre clairement l'«impatience» croissante de ce groupe : on ne compte plus que $20 \%$ des attentes dépassant 3 ans en 1988 , contre $54 \%$ en 1978 !

Par ailleurs, en 1988, les femmes les plus jeunes ont déclaré un délai nécessaire pour concevoir sensiblement inférieur à celui des femmes plus âgées, alors qu'on ne constatait guère de différences selon l'âge en 1978; ce résultat pourrait traduire aussi une impatience croissante dans les générations les plus récentes.

A l'inverse, d'autres méthodes de comparaison plus indirectes laisseraient plutôt penser qu'une partie au moins de l'évolution observée est réelle. Sans en donner ici le détail, mentionnons seulement qu'un 


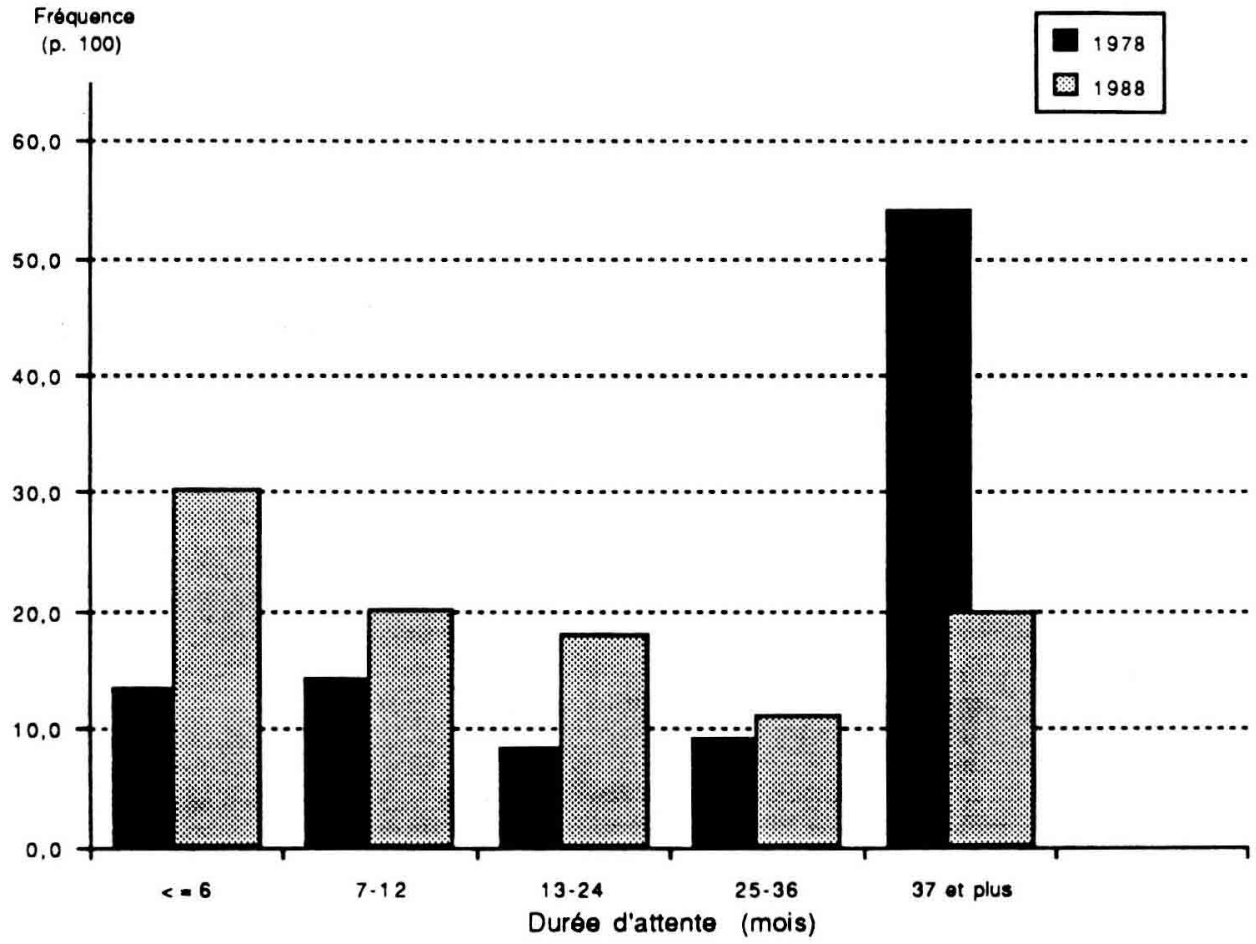

Fig 5. Répartition des anciennetés d'attente pour les femmes cherchant à concevoir au moment de l'enquête (INED 1978 et 1988).

de ces calculs conduirait à estimer à $30 \%$ environ la hausse de la proportion de couples hypofertiles entre les deux enquêtes, mais ce n'est qu'un résultat fragile qui devra être confirmé par d'autres analyses.

Pour l'heure, en tout cas, il semblerait donc que la "maîtrise de la reproduction" ait davantage progressé dans un sens que dans l'autre.

\section{RÉFÉRENCES}

Leridon $\mathrm{H}$, Charbit $\mathrm{Y}$, Collomb $\mathrm{P}$, Sardon JP, Toulemon $L$ (1987) La seconde révolution contraceptive. La régulation des naissances en France de 1950 à 1985. PUF, Cahier de I'INED 117.

Leridon $H$, Toulemon $L$ (1988) La contraception en France : pilule et stérilet. Population et Sociétés, 228 